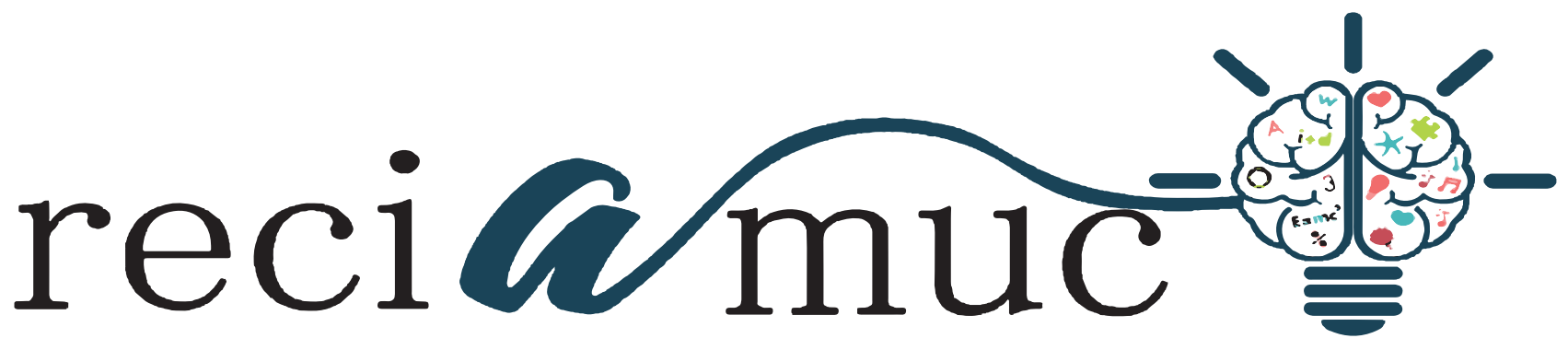

DOI: 10.26820/reciamuc/5.(4).noviembre.2021.48-59

URL: https://reciamuc.com/index.php/RECIAMUC/article/view/716

EDITORIAL: Saberes del Conocimiento

REVISTA: RECIAMUC

ISSN: 2588-0748

TIPO DE INVESTIGACIÓN: Artículo de Revisión

CóDIGO UNESCO: 32 Ciencias Médicas

PAGINAS: $48-59$

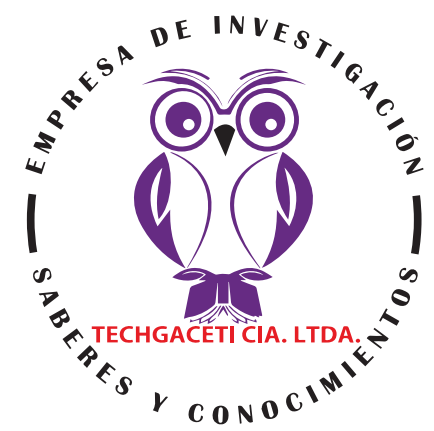

\title{
Manejo de shock séptico en unidad de cuidados intensivos
}

Management of septic shock in the intensive care unit Tratamento do choque séptico na unidade de terapia intensiva

\section{Karen Michelle Ruilova Gavilanes'; Karina Guadalupe Velasco Coellar2; Roberth Santiago Pienda Narvaez ${ }^{3}$ Diego Orlando Salazar Martínez ${ }^{4}$}

\section{RECIBIDO: 15/09/2021 ACEPTADO: 05/10/2021 PUBLICADO: 29/11/2021}

1. Médico General; Investigador Independiente; Quito, Ecuador; krenmr@gmail.com; iD https://orcid.org/00000001-5689-5067

2. Médico General; Investigador Independiente; Guaranda, Ecuador; karivelasco1510@hoymail.com; (iD https:// orcid.org/0000-0001-8632-7095

3. Médico General; Magister en Prevención de Riesgos Laborales; Ibarra, Ecuador; santypineda1992@hotmail. com; iD https://orcid.org/0000-0002-5451-1522

4. Médico General; Posgradista en Emergencias y desastres por la Universidad Central del Ecuador; diorsama@ gmail.com; iD https://orcid.org/0000-0002-7769-3082

\section{CORRESPONDENCIA}

Karen Michelle Ruilova Gavilanes

krenmr@gmail.com

\section{Quito, Ecuador}

@ RECIAMUC; Editorial Saberes del Conocimiento, 2021 


\section{RESUMEN}

En las salas de cuidados intensivos cuando se produce un shock séptico, el paciente se encuentra con una afección grave debido a una infección que se expande por todo el cuerpo desencadenando una peligrosa disminución en la presión arterial y un deficiente funcionamiento de los órganos. La sepsis es causada, por cualquier tipo de bacterias, hongos y, en ocasiones, por virus los cuales liberan toxinas provocando daño tisular. Sin embargo, la sepsis es común y casi siempre fatal por lo que se requiere de una intervención y manejo inmediato. El reconocimiento y el tratamiento temprano de esta afección han desafiado a los médicos y enfermeras de atención de emergencia y de cuidados intensivos. En tal sentido, es necesario que tanto el personal como los centros de salud, se encuentren al corriente con información actualizada y nuevas definiciones en el campo del manejo de la sepsis, con el objetivo de mejorar la condición del paciente. Aunque los reingresos hospitalarios después de la sepsis son comunes, los factores de riesgo asociados y el abordaje de los pacientes que sobreviven a este tipo de afección aún necesitan aclaración. La disfunción inmunológica causada por la sepsis / choque séptico es compleja, persistente, afecta los sistemas inflamatorios y antiinflamatorios y podría estar asociada con resultados a largo plazo. A través, de la siguiente revisión bibliográfica, se busca brindar información relevante y actualizada del manejo del shock séptico en las salas de cuidados intensivos.

Palabras clave: Shock séptico, cuidados intensivos, presión arterial, daño tisular.

\section{ABSTRACT}

In intensive care wards, when septic shock occurs, the patient encounters a serious condition due to an infection that spreads throughout the body, triggering a dangerous drop in blood pressure and poor organ function. Sepsis is caused by any type of bacteria, fungi and, sometimes, by viruses which release toxins causing tissue damage. However, sepsis is common and almost always fatal, requiring immediate intervention and management. Recognizing and treating this condition early have challenged emergency and intensive care physicians and nurses. In this sense, it is necessary that both the staff and the health centers are up to date with updated information and new definitions in the field of sepsis management, with the aim of improving the patient's condition. Although hospital readmissions after sepsis are common, the associated risk factors and management of patients who survive this type of condition still need clarification. Immune dysfunction caused by sepsis / septic shock is complex, persistent, affects inflammatory and anti-inflammatory systems, and could be associated with long-term outcomes. Through the following bibliographic review, we seek to provide relevant and updated information on the management of septic shock in intensive care rooms.

Keywords: Septic shock, intensive care, blood pressure, tissue damage.

\section{RESUMO}

Nas enfermarias de terapia intensiva, quando ocorre o choque séptico, o paciente encontra uma condição grave devido a uma infecção que se espalha por todo o corpo, desencadeando uma queda perigosa da pressão arterial e mau funcionamento dos órgãos. A sepse é causada por qualquer tipo de bactéria, fungo e, às vezes, por vírus que liberam toxinas causando danos aos tecidos. No entanto, a sepse é comum e quase sempre fatal, exigindo intervenção e tratamento imediatos. Reconhecer e tratar essa condição precocemente tem desafiado médicos e enfermeiras de emergência e de terapia intensiva. Nesse sentido, é necessário que tanto a equipe quanto os centros de saúde estejam atualizados com informações atualizadas e novas definições no campo do manejo da sepse, com o objetivo de melhorar o estado do paciente. Embora reinternações hospitalares após sepse sejam comuns, os fatores de risco associados e o manejo de pacientes que sobrevivem a esse tipo de condição ainda precisam de esclarecimento. A disfunção imunológica causada por sepse / choque séptico é complexa, persistente, afeta os sistemas inflamatório e antiinflamatório e pode estar associada a desfechos em longo prazo. Por meio da seguinte revisão bibliográfica, buscamos fornecer informações relevantes e atualizadas sobre o manejo do choque séptico em unidades de terapia intensiva.

Palavras-chave: Choque séptico, terapia intensiva, pressão arterial, danos aos tecidos. 


\section{Introducción}

La sepsis es común e incluso se considera mortal, lo que representa un importante problema de salud pública. Cada año, aproximadamente, "31 millones de personas sufren un episodio de sepsis. De estos, unos 6 millones de personas fallecen a causa de la sepsis" (OPS, 2019). En los países sub desarrollados la incidencia de sepsis es mayor y representa una de las causas principales de muerte maternal y neonatal. Sin embargo, hacer una estimación mundial resulta difícil debido a las limitantes en el diagnóstico.

Existe un consenso en que la incidencia está aumentando, impulsada por una población que envejece con múltiples comorbilidades, mayor uso de terapia inmunosupresora e intervenciones de alto riesgo. "Las estimaciones de mortalidad por sepsis oscilan entre el 27\% y el 36\%" (Kumar \& Taneja, 2011); sin embargo, el riesgo de muerte por sepsis ha ido disminuyendo en las últimas décadas a pesar del aumento de la incidencia, quizás debido a las mejoras en la atención.

Las Definiciones del Tercer Consenso Internacional para Sepsis y Choque Séptico (Sepsis-3) se publicó en febrero de 2016. Las nuevas definiciones están destinadas a mejorar la claridad de la atención clínica, epidemiología, mejora de la calidad e investigación. "Estas definiciones se han validado retrospectivamente mediante el uso de grandes bases de datos en EE. UU y Alemania" (Singer, Deutschman, \& Seymour, 2016).

La fisiopatología de la sepsis es difícil de comprender. Se produce una reacción fisiológica normal que pasa a ser infec- ción tanto proinflamatoria como antiinflamatoria, dando como resultado una disfunción orgánica potencialmente mortal.

La Campaña Sobreviviendo a la Sepsis (Surviving Sepsis Campaign (SSC)), ha sido una influencia clave en el cuidado de la sepsis y es una colaboración internacional única para la mejora de la calidad que fue establecida en 2002 por la Sociedad Europea de Medicina de Cuidados Críticos, el Foro Internacional de Sepsis y la Sociedad de Medicina de Cuidados Críticos (Rhodes, Evans, \& Alhazzani, 2017 ).

El objetivo de esta revisión es actualizar al lector sobre la última definición de sepsis y las implicaciones de los diferentes ensayos en aras de a ayudar a los profesionales médicos no intensivistas a reconocer los casos de sepsis y garantizar que el manejo de los pacientes sea adecuado y óptimo.

\section{Metodología}

El camino metodológico que caracteriza al presente artículo, se encuentra determinado por la presencia de argumentos de investigación caracterizados por ofrecer informaciones pertinentes al tema debidamente seleccionado, el mismo se apoya en las ideas dadas por lo citado por Ortiz y García (2018) para lograr eficiencia en una investigación es importante seleccionar el método que se convierte en una herramienta de recopilación de información determinada por momentos teóricos y prácticos".(p. 65)

En consecuencia, para dar continuidad al proceso investigativo se hace necesario identificar el método analítico como pieza fundamental que gira las acciones básicas encargadas de encaminar 
el hecho científico bajo una valoración precisa y coherente de los aspectos que identifican previamente el fenómeno en estudio. Tal como lo define Ortiz y García (2018) es aquel método que "consiste en la desmembración de un todo descomponiéndolo en sus elementos para observar las causas, naturaleza y efectos" (p.65). De allí, que su introducción en este artículo, hace posible desglosar el contenido temático en partes esenciales que al sumarse logran construir básicamente las apreciaciones generales que el autor considera pertinente.

\section{Tipo de Investigación}

Una vez seleccionado el método se hace necesario recurrir a la ubicación dentro del campo científico al artículo, lo que asegura su relación con los estudios documentales visto por Zambrano (2018) "como la recopilación de informaciones provenientes de materiales impresos, audiovisuales, tecnológicos entre otras, para expresar nuevas ideas acerca de un tema en particular" (p.33). Es decir, mediante este tipo de investigación, se puede replantear nuevas valoraciones referidas a un contenido en particular, que hace posible conjugar diferentes interpretaciones para finalmente complementar sus beneficios dentro de una teoría en particular.

\section{Fuentes Documentales}

La conducción de la dinámica científica amerita para ser viable a un proceso veraz, el complemento de aquellas fuentes documentales que le dan oportunidad de encontrar de forma precisa el respectivo dominio de un fenómeno de acuerdo con sus características especiales que le asignan un conocimiento enmarcado en una realidad previa. En tal sen- tido, Zambrano (2018) destaca que las fuentes documentales "es la suma de técnicas que permiten orientar el trabajo desde una perspectiva cotidiana para cumplir con los diferentes procedimientos mediante explicaciones" (p.35). Por lo tanto, su aplicación en el estudio, permite asegurar con antelación, la revisión de diferentes documentos propios y relacionados con el tema, para luego elaborar su contenido que quedará como representación del fenómeno en estudio.

\section{Técnicas para la Recolección de la In- formación}

Con el fin de darle operatividad a la investigación, se busca primeramente plantear la definición de técnica dada por Zambrano (2018) "son los diferentes procedimientos para el aprovechamiento científico de los elementos de la naturaleza y sus derivados" (p.35). Según lo citado, permite interpretar que darle la respectiva complejidad al análisis que se busca del tema previo, se hace necesario adecuar las apreciaciones en función a los procedimientos que den cabida a un mayor aprovechamiento de los elementos presente en la naturaleza del tema seleccionado.

De este modo, durante la fase de recolección de la información, se llevaron a cabo acciones especiales que dieron cabida a incorporar las técnicas de fuentes de información definidas por Zambrano (2018) "como aquellas herramientas básicas para las investigaciones documentales que conllevan al uso óptimo y racional de los recursos documentales" (p.35). En consecuencia mediante su utilización, se puede organizar los diferentes contenidos disponibles en la web considerando que aquella heren- 
cia de la globalización permite acceder a mayor y mejor información a través de las herramientas tecnológicas. El motor de búsqueda ha sido herramientas académicas de la web que direccionan específicamente a archivos con validez y reconocimiento científico, descartando toda información no confirmada o sin las respectivas referencias bibliográficas.

\section{U}

\section{(1) Resultados}

La incidencia y la prevalencia de la sepsis han aumentado a nivel mundial durante los últimos años.

La sepsis es la causa más frecuente de ingreso en una unidad de cuidados intensivos $(\mathrm{UCl})$, la causa más común de muerte en la $\mathrm{UCl}$ y de reingreso hospitalario en los supervivientes de sepsis. Adicionalmente, se ha informado como la última vía hacia la muerte por infección (Kissoon, Daniels, Van der Poll, \& Finfer, 2016).

El aumento progresivo del número de supervivientes de sepsis y shock séptico "enfatiza las consecuencias a largo plazo de la sepsis, como disfunción cognitiva y discapacidades funcionales, morbilidad psiquiátrica, disminución de la calidad de vida relacionada con la salud, hospitalización no planificada readmisiones y mortalidad tardía" (Sun, Netzer, \& Small, 2016).

De acuerdo con Rhodes, Evans, \& Alhazzani, (2017 ) el SSC ha desarrollado pautas basadas en evidencia para el manejo de la sepsis, en conjunto con intervenciones para cambiar la práctica de cabecera. A continuación se muestran las recomendaciones claves de acuerdo a las directrices actualizadas en el 2016.
EI UK Sepsis Trust ha desarrollado una iniciativa llamada "Sepsis Six" diseñada para facilitar la entrega del paquete de reanimación SSC. "El paquete sepsis seis está diseñado para completarse dentro de 1 hora e incluye medidas sencillas para evaluación, reanimación y estratificación de riesgos, que pueden ser implementados al lado de la cama por enfermeras y médicos" (Kissoon, Daniels, Van der Poll, \& Finfer, 2016). A continuación se presenta un cuadro donde se observa lo planteado por el autor. 
Tabla 1. Clave de la Campaña Sobrevivir a la sepsis 2016 recomendaciones

Los antibióticos intravenosos deben iniciarse en el plazo de 1 hora luego del reconocimiento de sepsis y debe incluir terapia combinada

(al menos dos clases de antibióticos para cubrir una sospecha de patógeno) para pacientes con shock séptico.

La terapia combinada no debe usarse de forma rutinaria para pacientes sin shock.

Los pacientes con hipoperfusión deben recibir al menos $30 \mathrm{ml} /$

$\mathrm{kg}$ de cristaloide intravenoso dentro de las 3 horas y deben ser re-evaluados con frecuencia.

Para los pacientes que requieren vasopresores, el objetivo inicial La presión arterial (MAP) debe ser de $65 \mathrm{~mm} \mathrm{Hg}$.

La noradrenalina es la primera opción para los pacientes que necesitan vasopresores. Se puede agregar vasopresina o epinefrina.

En pacientes que permanecen inestables, se recomienda la dobutamina.

Se sugiere hidrocortisona intravenosa (200 mg / día) para pacientes hemodinámicamente inestables a pesar de los líquidos y vasopresores.

La transfusión de sangre debe reservarse para pacientes con una concentración de hemoglobina de $<7,0 \mathrm{~g} / \mathrm{dL}$, excepto en circunstancias especiales como hemorragia, miocardio e isquemia. Se deben administrar plaquetas si el recuento de plaquetas

$$
\begin{gathered}
\text { es }<10000 / \mathrm{mm} 3 \text { o }<20000 / \mathrm{mm} 3 \\
\text { con sangrado. }
\end{gathered}
$$

El bicarbonato de sodio no debe usarse en la mayoría de los pacientes. con $\mathrm{pH} \geq 7,15$.

Fuente: (Rhodes, Evans, \& Alhazzani, 2017).

Tabla 2. Paquete de tratamiento de Sepsis Seis que debe completarse en 1 hora.

Administrar oxígeno para mantener la $\mathrm{SpO} 2$ a $>94 \%$.

Tomar hemocultivos y considerar fuente infecciosa.

Administrar antibióticos por vía intravenosa.

Considere la reanimación con líquidos por vía intravenosa.

Verifique los lactatos seriados.

Inicie la medición de la producción de orina por hora.

Fuente: (Kissoon, Daniels, Van der Poll, \& Finfer, 2016) 
La terapia temprana dirigida por objetivos (EGDT) de acuerdo a sus siglas en ingles "es un protocolo de reanimación cuantitativa que establece objetivos de reanimación con el fin de restaurar el tejido perfusión en pacientes con shock séptico" (Rivers, Nguyen, \& Havstad, 2001). Fue primero probado con éxito por Rivers junto a sus colaboradores en 2001 y formó la base del paquete de 6 horas del SSC.

Más recientemente, tres grandes multicéntricos estudios los controlados aleatorizados, como los Protocolizados Cuidado del shock séptico temprano (ProCESS), la Reanimación de Australasia en la evaluación de la sepsis (ARISE) y el ensayo Protocolised Management in Sepsis (ProMISe) no demostraron beneficio de resultado de EGDT en comparación con la habitual atención en pacientes con shock séptico (Mouncey, Osborn, \& Power, 2015).

Sin embargo, respecto al nuevo SSC las directrices restan importancia a la protocolización de la atención y monitorización invasiva. "Ellos recomiendan que los pacientes deben ser reevaluados con frecuencia por los médicos" (Rhodes, Evans, \& Alhazzani, 2017).

Los datos de un estudio de cohorte prospectivo de SSC "mostró que el cumplimiento de los paquetes SSC condujo a una reducción del riesgo relativo de mortalidad del 25\%" (Levy, Rhodes, \& Phillips, 2015). En tal sentido, la revisión de la atención combinada para el shock séptico ha demostrado mejoras constantes en la supervivencia. Por lo tanto, aunque la efectividad de EGDT ahora es incierta, hay pocas dudas de que los paquetes de sepsis mejoran los resultados.

\section{Reconocimiento de la sepsis}

La ventana entre el inicio y la identificación de la sepsis es casi siempre cuando se producen retrasos importantes en el tratamiento y cuando el tratamiento adecuado es crucial. "El reconocimiento rápido y la reanimación de los pacientes con sepsis es, por tanto, clave para el tratamiento eficaz de la sepsis" (Daniels, 2011). Por lo tanto, los pacientes en deterioro con una puntuación de alerta temprana elevada (como una puntuación de alerta temprana agregada elevada de 5 o superior) deben someterse a pruebas de detección de infección.

Cuando se enfrenta a un paciente con sepsis, las evaluaciones iniciales para identificar la hipoperfusión de órganos terminales e identificar la fuente de las infecciones es importante. "Solo se deben administrar antibióticos inmediatamente cuando es probable que haya demoras en la obtención de muestras por la cultura; sin embargo, casi siempre es posible obtener sangre cultivos antes de administrar antibióticos" (Daniels, 2011).

Las evaluaciones para el diagnóstico de hipoperfusión de órganos terminales incluyen:

- Hemograma completo

- Perfil de coagulación, con tiempo de protrombina e INR

- Urea y electrolitos

- Pruebas de función hepática

- Lactato sérico

- Medición de gases en sangre arterial. 
Estas evaluaciones deben realizarse y los resultados deben revisarse sin retraso. "La mayoría de las áreas clínicas tienen acceso a laboratorio máquinas de medición de lactato o de gases en sangre arterial capaces de proporcionando un resultado rápido de lactato arterial o venoso" (Rivers, Nguyen, \& Havstad, 2001).

\section{Manejo clínico inicial de la sepsis}

Los objetivos terapéuticos en el manejo de la sepsis son mejorar oxigenación, la perfusión tisular y proporcionar antimicrobianos como terapia con cobertura adecuada contra el organismo causante.

\section{Antibióticos}

EI SSC ha abogado por la terapia antibiótica temprana desde su inicio, y la recomendación ha sido apoyada por evidencia convincente. "Las guías de SSC actualizadas de 2016 recomiendan que se administren antibióticos intravenosos dentro de 1 hora de reconocimiento de la sepsis" (Rhodes, Evans, \& Alhazzani, 2017).

\section{Líquidos intravenosos}

\section{Tipo de fluido}

La reanimación inicial con líquidos debe realizarse con cristaloides. No hay evidencia convincente que sugiera la superioridad de ninguna alternativa. Las soluciones coloides pueden estar asociadas con un mayor riesgo de insuficiencia renal aguda y se ha observado un beneficio marginal para reanimación con soluciones que contienen albúmina.40-42 Los cristaloides son más baratos y más fáciles de conseguir. "Así SSC recomienda usar cristaloides inicialmente y que las soluciones que contienen albúmina se pueden usar en pacientes que han recibido grandes volúmenes de cristaloides" (Levy, Rhodes, \& Phillips, 2015).

\section{Fuente de control}

El control de la fuente implica medidas tomadas para eliminar focos de infección y controlar la contaminación en curso restaurando la anatomía y las funciones premórbidas. Según Marshall, Maier, \& Jimenez, (2004) "se recomienda que esto se lleve a cabo dentro de las primeras 12 horas después del diagnóstico, y el procedimiento menos invasivo debe ser usó". Esto puede incluir "el drenaje de acumulaciones de líquido infectado, desbridamiento de tejido sólido infectado y extracción de dispositivos y cuerpos extraños, incluidos dispositivos de acceso intravascular o cirugía" (Marshall, Maier, \& Jimenez, 2004).

\section{Monitoreo de lactato}

Los ensayos han demostrado que "los pacientes que eliminan los niveles elevados de lactato han mejorado los resultados, sin embargo, cuanto más lenta es la eliminación, el resultado no es tan bueno" (Jansen, Van Bommel, \& Schoonderbeek, 2010). Por lo tanto, la reanimación puede lograr la normalización del lactato sérico, obtenido por serie venosa. En un entorno que no sea de cuidados intensivos, volver a medir el lactato puede identificar aquellos que no responden al líquido intravenoso de resucitación.

\section{Soporte de la vía aérea y oxígeno}

Una de las prioridades en el manejo de un paciente con una enfermedad es estabilizar las vías respiratorias, con el objetivo de asegurar una adecuada oxigenación de los tejidos. 
El uso empírico de oxígeno de alto flujo ya no es recomendado en el paquete Sepsis Six. El oxígeno debe ser utilizado para mantener las saturaciones > 94\%, a menos que haya un antecedente o sospecha clínica de enfermedad pulmonar obstructiva crónica, o enfermedad e insuficiencia respiratoria tipo II (MarshaII, Maier, \& Jimenez, 2004).

\section{Tratamiento clínico adicional de la sepsis}

Los pacientes en los que los objetivos fisiológicos no se pueden alcanzar con líquido intravenoso solo, pueden requerir un tratamiento adicional para optimizar la perfusión de los órganos terminales. En realidad, esto generalmente se proporcionará en un entorno de cuidados intensivos.

\section{Vasopresores e inotrópicos}

Los vasopresores aumentan la presión arterial al aumentar la resistencia vascular periférica. "En pacientes que permanecen hipotensos a pesar de una adecuada reanimación con líquidos, o en aquellos que desarrollan edema pulmonar cardiogénico, se debe utilizar un vasopresor. Sin embargo, el uso de vasopresores y los introptos deben limitarse a cuidados intensivos" (Rhodes, Evans, \& Alhazzani, 2017).

\section{Corticoesteroides}

La hidrocortisona como terapia adyuvante "puede tener un papel en los pacientes con shock séptico que no logran una PAM adecuada a pesar de los líquidos intravenosos y vasopresores adecuados" (Levy, Rhodes, \& Phillips, 2015) Sin embargo, su uso indiscriminado en todos los pacientes con shock séptico no mostró ningún beneficio en el gran es- tudio multicéntrico CORTICUS (Sprung, Annane, \& Keh, 2008).

\section{Transfusión de hemoderivados}

Los pacientes con sepsis deben recibir una transfusión de glóbulos rojos cuando la hemoglobina cae por debajo de $<7$ $\mathrm{g} / \mathrm{dL}$. "Esto sigue la evidencia de un gran ensayo multicéntrico que compara un restrictivo ( $<7 \mathrm{~g} / \mathrm{dL}$ ) con un umbral de transfusión liberal $(<9 \mathrm{~g} / \mathrm{dL})$ " (Marshall, Maier, \& Jimenez, 2004). Los pacientes con sangrado, isquemia miocárdica o hipoxia severa son excepciones y deben transfundirse a umbrales de hemoglobina más altos. No se debe utilizar plasma fresco congelado para corregir los datos documentables coagulopatía a menos que haya hemorragia intercurrente o invasiva.

\section{Control de glucosa}

Hay una falta de evidencia clara con respecto a los objetivos para un óptimo control de la glucosa con insulina en pacientes con sepsis, en parte debido a la gran variedad de factores y variaciones del paciente en los protocolos de glucosa de varios ECA (Wilson, Weinreb, \& Hoo, 2007).

Cada vez es más claro a partir de una serie de metanálisis que el objetivo para el control intensivo de la glucosa en sangre $(<6,1 \mathrm{mmol} / \mathrm{L})$ confiere sin beneficio de supervivencia. De hecho, el estudio más sólido (NICESUGAR) que comparó el control intensivo de la glucosa en sangre con el control estándar de insulina, utilizando un límite de glucosa en sangre para la terapia con insulina de $10 \mathrm{mmol}$ / L, mostró un aumento de la mortalidad en el grupo de insulina intensiva (Finfer \& Chittock, 2009). 
Por lo tanto, SSC recomienda intervención (con un protocolo de insulina) para mantener la glucosa en un valor de corte de $10 \mathrm{mmol} / \mathrm{L}$ en sepsis y para evitar hipoglucemias y fluctuaciones rápidas de glucosa. "La glucosa sérica debe ser monitoreada cada 1 a 2 horas hasta que sea estable, y cada 4 horas después" (Levy, Rhodes, \& Phillips, 2015).

\section{Conclusión}

Si bien existe un conjunto de pruebas cada vez mayor que informa el manejo óptimo de la sepsis en entornos de cuidados intensivos, el personal médico seguirá desempeñando un papel clave en la identificación y los tratamientos precoces de estos pacientes. En tal sentido, es esencial que todo el personal médico esté bien educado en el reconocimiento y manejo de la sepsis, y que los sistemas estén en el lugar para garantizar que esto se haga de manera confiable.

A pesar del desempeño en crear definiciones y una guía clara que dicta las mejores prácticas en el manejo de la sepsis, siguen existiendo barreras considerables para implementar la orientación de la SSC. Se debe tener presente, que el primer paso crucial es el reconocimiento de septicemia. El proceso de hacer coincidir parámetros anormales con el diagnóstico de una infección y, posteriormente, de una sepsis puede ser difícil y puede provocar retrasos en el inicio de un tratamiento óptimo.

El shock requiere de evaluaciones, exámenes de laboratorio y tratamiento de cuidados intensivos, lo que puede introducir más retrasos en un hospital de alta demanda. Además, la evaluación y el tratamiento avanzado de los pacientes con sepsis, requieren de un entorno de cuidados intensivos. Por lo tanto, para la atención eficaz de la sepsis, es necesaria una coordinación entre el diagnóstico, el tratamiento y el tiempo de detección o reconocimiento de dicha afección. 


\section{Bibliografía}

Daniels, R. (2011). Surviving the first hours in sepsis: getting the basics right (an intensivist's perspective). J Antimicrob Chemother, 66(2), 11-23. doi:10.1093/jac/dkq515.

Finfer, S., \& Chittock, D. (2009, Mar 26). Intensive versus conventional glucose control in criticaIly ill patients. N Engl J Med, 360(13), 1283-97. doi:10.1056/NEJMoa0810625

Jansen, T., Van Bommel, J., \& Schoonderbeek, F. (2010). Early lactate-guided therapy in intensive care unit patients: a multicenter, open-label, randomized controlled trial. Am J Respir Crit Care Med, 182(6), 752-61. doi:10.1164/rccm.200912-19180C

Kissoon, N., Daniels, R., Van der Poll, T., \& Finfer, S. (2016, Jun). Sepsis - the final common pathway to death from multiple organ failure in infection. Crit Care Med, 44 (6), e446. doi:10.1097/ CCM.0000000000001582.

Kumar, G., \& Taneja, A. (2011). Nationwide trends of severe sepsis in the 21st century (2000-2007). Chest 2011. Chest, 140, 1223-31. doi:DOI:10.1378/ chest.11-0352

Levy, M., Rhodes, A., \& Phillips, G. (2015, Jan). Surviving Sepsis Campaign: association between performance metrics and outcomes in a 7.5-year study. Crit Care Med, 43(1), 312. doi:doi: 10.1097/ CCM.0000000000000723.

Marshall, J., Maier, R., \& Jimenez, M. (2004). Source control in the management of severe Sepsis and septic shock: an evidence-based review. Crit Care Med, 32(11), 513-26.

Mouncey, P., Osborn, T., \& Power, G. (2015). Trial of early, goal-directed resuscitation for septic shock. N Engl J Med, 372, 1301-11. doi:10.1056/NEJMoa1500896

OPS. (2019, Oct 18). Organización Panamericana de la Salud. Retrieved Nov 11, 2021, from https:// www3.paho.org/hq/index. php?option=com_content\&view=article\&id=14278: sepsis-general-information\&ltemid $=72260 \&$ lang $=$ es

Ortíz, F., \& García, C. (2018). Metodología de la Investigación. México: Vergara.

Rhodes, A., Evans, L., \& Alhazzani, W. (2017, Mar). Surviving Sepsis Campaign: International Guidelines for Management of Sepsis and Septic Shock: 2016. Intensive Care Med, 43(3), 304-377. doi:10.1007/s00134-017-4683-6.

Rivers, E., Nguyen, B., \& Havstad, S. (2001, Nov
8). Early goal-directed therapy in the treatment of severe Sepsis and septic shock. N Engl J Med, 345(19), 1368-77. doi:10.1056/NEJMoa010307.

Singer, M., Deutschman, C., \& Seymour, C. (2016). The Third International Consensus Definitions for Sepsis and Septic Shock (Sepsis-3). JAMA, 315, 801-10. doi:doi:10.1001/jama.2016.0287

Sprung, C., Annane, D., \& Keh, D. (2008, Jan 10). Hydrocortisone therapy for patients with septic shock. N Engl J Med, 358(2), 111-24. doi:10.1056/ NEJMoa071366

Sun, A., Netzer, G., \& Small, D. (2016). Association between index hospitalization and hospital readmission in sepsis survivors. Crit Care Med, 44(3), 478-487. doi:10.1097/CCM.0000000000001464.

Wilson, M., Weinreb, J., \& Hoo, G. (2007). Intensive insulin therapy in critical care: a review of 12 protocols. Diabetes Care, 30(4), 1005-11. doi:10.2337/ dc06-1964.

Zambrano, P. (2018). Método Científico. México: Fondo Educativo Interamericano 


\section{CITAR ESTE ARTICULO:}

Ruilova Gavilanes, K. M., Velasco Coellar, K. G., Pienda Narvaez, R. S., \& Salazar Martínez, D. O. (2021). Manejo de shock séptico en unidad de cuidados intensivos. RECIAMUC, 5(4), 48-59. https://doi.org/10.47464/reciamuc/5.(4). noviembre.2021.48-59 MERCIAL-COMPARTIRIGUAL 4.0. 